\title{
An Intercomparison Between NPL (India) and NIST (USA) Pressure Standards in the Hydraulic Pressure Region up to $26 \mathrm{MPa}$
}

\section{J. K. N. Sharma and Kamlesh K. Jain}

National Physical laboratory, New Delhi, India

and

C. D. Ehrlich, J. C. Houck, and D. B. Ward

National Institute of Standards and Technology, Gaithersburg, MD 20899-0001
Results are presented of an intercomparison of pressure measurements between the National Physical Laboratory (NPL), India, and the National Institute of Standards and Technology (NIST), USA, using piston gauge pressure standards over the range $6 \mathrm{MPa}$ to $26 \mathrm{MPa}$. The intercomparison, using the NPL piston gauge pressure standard, with a nominal effective area of $8.4 \times 10^{-5} \mathrm{~m}^{2}$, and the NIST piston gauge pressure standard, with a nominal effective area of $2.0 \times 10^{-5} \mathrm{~m}^{2}$, was carried out at the NPL.

The intercomparison data obtained show a relative difference of $1 \times 10^{-6}$ in the zeropressure effective area $\left(A_{0}\right)$ of the NPL

\begin{abstract}
standard as obtained by the NIST standard. At $6 \mathrm{MPa}$ the relative difference in effective areas is $3.5 \times 10^{-6}$; at the full scale pressure of $26 \mathrm{MPa}$, the relative difference is $12 \times 10^{-6}$. These differences are in excellent agreement with the statements of uncertainty of the respective standards as obtained from the primary standards of these two laboratories.
\end{abstract}

Key words: hydraulic pressure, piston gauge; pressure.

Accepted: July 14, 1994

\section{Introduction}

Piston gauges [1] and liquid column manometers [2] are widely used instruments for the most accurate measurement of pneumatic pressure in the near-atmospheric pressure region ( $10 \mathrm{kPa}$ to $1 \mathrm{MPa})$. Piston gauges are also used to measure pneumatic pressures to over 100 $\mathrm{MPa}$, and hydraulic pressures from as low as $1 \mathrm{MPa}$ to over I GPa. In a typical piston gauge, a cylindrical piston rotates in a closely-fitted cylinder. The pressure is derived from the known downward gravitational force on the piston and weights that is balanced by an upward force generated by the action of the system pressure on a known area when the piston is floating at its reference position. The uncertainty with which a pressure measurement can be made using a piston gauge then depends on the uncertainties with which measurements of both the downward force and the effective area of the piston-cylinder assembly can be made.
The elastic deformation of the piston-cylinder assembly is usually negligible in the atmospheric pressure range. The uncertainty in the evaluation of the effective area at low pressure [3] is mainly limited by the uncertainty with which absolute dimensional measurements can be made of the piston and cylinder. Recent studies [4] have shown that the effective areas of large-diameter $(35 \mathrm{~mm})$, atmospheric-pressure range piston-cylinder assemblies may have significant distortion coefficients, at the 6 parts in $10^{6}$ level. Even so, effective areas of these gauges obtained by dimensional measurements and incorporating theoretical distortion coefficients based on simple elastic theory are found to agree to within 10 parts in $10^{6}$ with values obtained by crossfloat against either manometers or standard gauges having known pressure dependence of the effective area. 
At higher pressures, besides the dimensional uncertainty, there is additional uncertainty in determining the effective area of a piston gauge due to the distortion of the piston and cylinder assembly. Compounding the problem, higher-pressure pistons and cylinders typically have relatively small diameters, so that a given dimensional uncertainty results in a relatively larger uncertainty in both the low-pressure effective area $\left(A_{0}\right)$ and the distortion coefficient (b) of the gauge [5].

Close agreement of experimentally-determined pressure-dependent effective areas, using different techniques within a metrological laboratory, creates confidence in the measurements. To add further confidence in pressure measurement, international intercomparisons are often performed. Such intercomparisons can establish uniformity of measurements and mutual compatibility of standards, and reveal possible systematic errors or reaffirm the uncertainty within which the laboratory can make relative pressure measurements.

While the results of international intercomparisons of piston gauges in the pneumatic pressure region $[6,7]$ up to $10 \mathrm{MPa}$, and at hydraulic pressures [8-12] up to 500 $\mathrm{MPa}$, are reported in the literature, there is relatively little such intercomparison in the lower hydraulic pressure region. With this in mind, a series of pressure comparison measurements in the hydraulic hydrostatic pressure region up to $26 \mathrm{MPa}$ was carried out between NPL (India) and NIST (USA). Direct piston gauge crossfloats used to accomplish the comparison were performed at NPL, and the results are reported here.

\section{Description and Metrological Charac- teristics of the Standards}

\subsection{The NPL Transfer Standard}

The NPL transfer standard piston gauge that was used for these measurements, denoted NPL-28, is equipped with a reentrant type piston-cylinder system capable of measuring a full-scale pressure of $28 \mathrm{MPa}$. A schematic representation of the NPL piston gauge is shown in Fig. 1. The piston is rotated to relieve friction by a pulley coupled to a dc motor. To minimize thermal problems the motor is mounted at a distance of $300 \mathrm{~mm}$ from the piston-cylinder assembly. The effective area of NPL-28 was determined by dimensional measurements and also by crossf loating over the range $5 \mathrm{MPa}$ to $26 \mathrm{MPa}$ against another NPL piston gauge of $100 \mathrm{MPa}$ full scale pressure, denoted NPL-100. NPL-100 in turn was calibrated against the NPL controlled clearance primary pressure standard. The other parameters associated with NPL-28 are given in Table 1. Figure 2 shows the residuals from the best linear fit of the effective area $A_{\mathrm{e}}$ of NPL-28 as a function of the nominal applied pressure, as obtained using NPL-100 as the standard. The best linear fit of the model $A_{\mathrm{e}}=A_{0}(1+b p)$ is obtained when $A_{0}=8.400$ $423 \times 10^{-5} \mathrm{~m}^{2}$ and $b=-1.62 \times 10^{-12} \mathrm{~Pa}^{-1}$.

The $3 \sigma$ standard deviation of the $A_{0}$ coefficient is $\left(6 \times 10^{-6)} A_{0}\right.$. The $3 \sigma$ overall uncertainty of $A_{\mathrm{c}}$ of NPL-28 as obtained during calibration by NPL- 100 is $\left(88 \times 10^{-6)} A_{\mathrm{c}}\right.$.

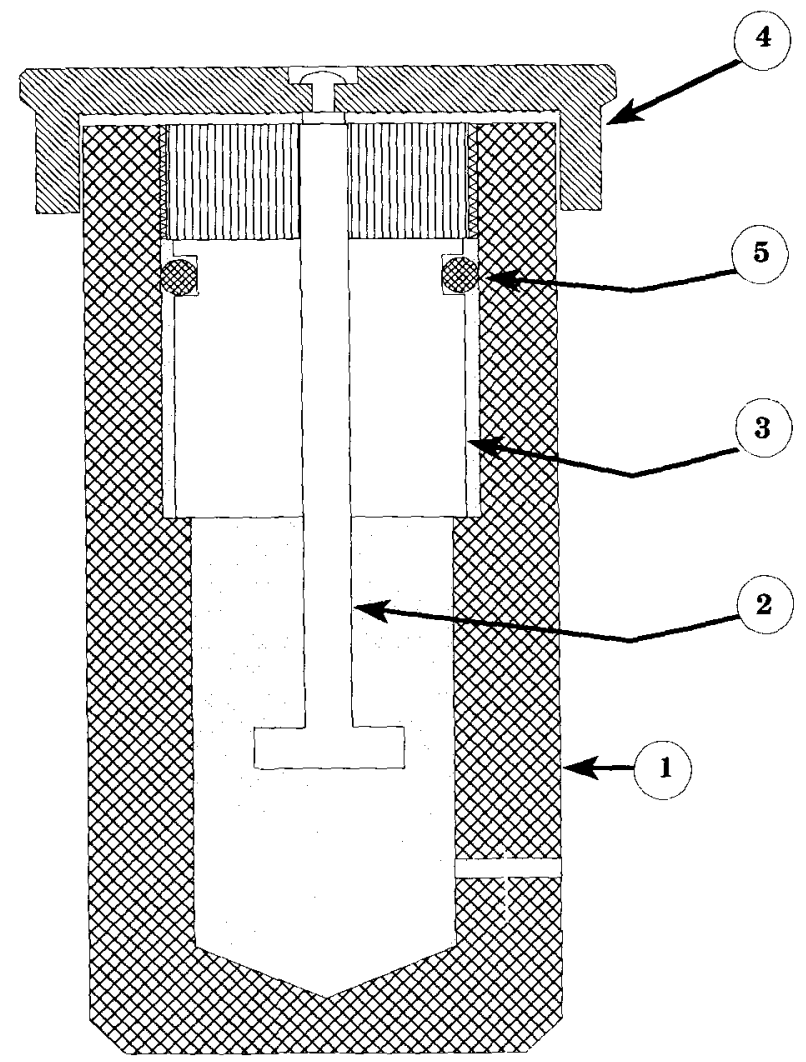

Fig. 1. A schematic cross-sectional view of the measuring system of the NPL piston gauge standard NPL-28: (1) pressure column, (2) piston, (3) cylinder assembly, (4) weight table (5) O-rings.

\subsection{The NIST Transfer Standard}

The NIST transfer standard piston gauge, denoted NIST-45, is equipped with a simple-type piston-cylinder assembly having a full pressure range of $50 \mathrm{MPa}$. The piston is rotated by an oval-shaped pulley coupled to a dc motor mounted at a distance from the piston-cylinder in order to minimize the heat transferred from the motor to the piston and cylinder during operation. The effective area and the pressure coefficient of the piston-cylinder assembly of NIST-45 were obtained at NIST [13] by calibrating NIST-45 against primary controlled clearance piston gauge NIST-27, which has a full pressure range of $28 \mathrm{MPa}$. Figure 3 shows the residuals of the effective area of NIST- 45 from the best fit of the model $A_{\mathrm{c}}=A_{0}(1+b p)$ where $A_{0}=1.961 \quad 191 \times 10^{-5} \mathrm{~m}^{2}$ and $b=9.85 \times 10^{-13} \mathrm{~Pa}^{-1}$. The $3 \sigma$ uncertainty of $A_{\mathrm{c}}$ of NIST45 as obtained during calibration by NIST-27 is ${ }^{(35} \times 10^{-6)} A_{\mathrm{e}}$. 
Table 1. Description and metrological parameters of the pistoncylinder assemblies used in the pressure comparison measurements

\begin{tabular}{|c|c|c|}
\hline Piston gauge designation & NPL-28 & NIST-45 \\
\hline Piston-cylinder (type) & Reentrant & Simple \\
\hline Full scale pressure (MPa) & 28 & 50 \\
\hline Piston material & Tungsten carbide & Tungsten carbide \\
\hline Cylinder material & Tungsten carbide & Tungsten carbide \\
\hline Fluid & Spinesstic $22^{\mathrm{a}}$ & Spinesstic $22^{a}$ \\
\hline $\begin{array}{l}\text { Coefficient of thermal } \\
\text { expansion for piston }\left({ }^{\circ} \mathrm{C}^{-1}\right)\end{array}$ & $4.5 \times 10^{-6}$ & $4.5 \times 10^{-6}$ \\
\hline $\begin{array}{l}\text { Coefficient of thermal } \\
\text { expansion for cylinder }\left({ }^{\circ} \mathrm{C}^{-1}\right)\end{array}$ & $4.5 \times 10^{-6}$ & $4.5 \times 10^{-6}$ \\
\hline $\begin{array}{l}\text { Effective area at } \\
\text { atmospheric pressure and } \\
\text { at } 23^{\circ} \mathrm{C}\left(\mathrm{m}^{2}\right)\end{array}$ & $8.400423 \times 10^{-5}$ & $1.961191 \times 10^{-5}$ \\
\hline Distortion coefficient $\left(\mathrm{Pa}^{-1}\right)$ & $-1.62 \times 10^{-12}$ & $9.85 \times 10^{-13}$ \\
\hline $\begin{array}{l}\text { Estimated total relative } \\
\text { uncertainty }(3 \sigma) \text { of the } \\
\text { effective area, } \Delta A_{\mathrm{d}} / A_{\mathrm{c}}\end{array}$ & $88 \times 10^{-6}$ & $35 \times 10^{-6}$ \\
\hline
\end{tabular}

${ }^{a}$ Certain commercial equipment, instruments, or materials are identified in this paper to foster understanding. Such identification does not imply recommendation or endorsement by the National Institute of Standards and Technology, nor does it imply that the materials or equipment identified are necessarily the best available for the purpose.

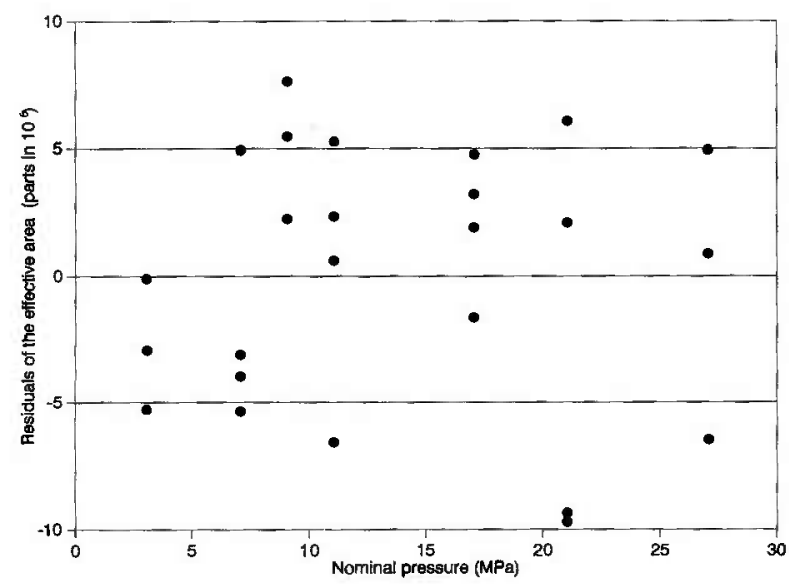

Fig. 2. Residuals of measured values of the effective area $\left(A_{\mathfrak{c}}\right)$ of PL-28 from the best linear fit of the model $A_{\mathrm{t}}=A_{0}(1+b p)$, where $A_{0}=8.400423 \times 10^{-5} \mathrm{~m}^{2}$ and $b=-1.62 \times 10^{-12} \mathrm{~Pa}^{-1}$, obtained when calibrated by the NPL standard (NPL-100).

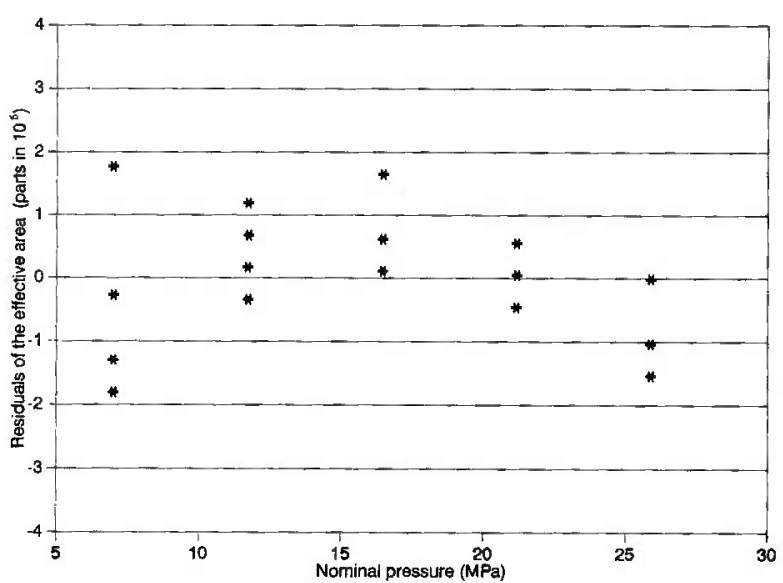

Fig. 3. Residuals of measured values of the effective area $\left(A_{\mathrm{e}}\right)$ of the NIST piston gauge standard NIST-45 from the best linear fit of the model $A_{c}=A_{0}(1+b p)$, where $A_{0}=1.961191 \times 10^{-5} \mathrm{~m}^{2}$ and $b=9.85 \times$ $10^{-13} \mathrm{~Pa}^{-1}$, obtained when calibrated by the NIST primary standard (NIST-27) used in the controlled clearance gauge mode.

\section{Experimental Procedure}

The piston gauges used were kept on a heavy nonmagnetic stainless steel base to minimize vibration and magnetic effects. All measurements were made in an environment which provided stable temperature conditions of $(23 \pm 1)^{\circ} \mathrm{C}$. The temperature of NPL-28 was measured within $0.1^{\circ} \mathrm{C}$ by a mercury-in-glass thermometer placed near the pressure column. The temperature of the NIST transfer standard was measured with a platinum resistance thermometer (PRT) attached near the piston, and its output was read with an autoranging digital multimeter having a resolution of $2 \mathrm{~m} \Omega$ corresponding to a temperature resolution of $0.005^{\circ} \mathrm{C}$.

The intercomparison between NPL-28 and NIST-45 was carried out using the well-established crossfloat method [1]. Before the crossfloat, both piston gauges were leveled to ensure the verticality of their axes, and the systems were checked for leaks to the full scale pressure of $28 \mathrm{MPa}$. The piston gauges were loaded with the weights calculated to generate the desired pressure, and were then pressurized to float at their reference levels. The gauges were then isolated from the rest of the pressurizing system, and subsequently from each other, by closing the isolation valves provided in the pressure line and between the gauges. The position and fall rate of both pistons were measured using the output of an electronic displacement transducer recorded on a strip chart recorder. By adjusting the fractional weights on NIST-45, which was generating comparatively lower pressures, crossfloat equilibrium was achieved, as determined when both gauges had the same respective fall 
rates independent of whether the isolation valve between the gauges was closed or open. The pressure was then increased to the next higher step, as discussed later, and the procedure was repeated, up to the pressure of 26 $\mathrm{MPa}$. A period of about $30 \mathrm{~min}$ between two successive pressures was found adequate to allow the system to return to equilibrium, and about $10 \mathrm{~min}$ was required to repeat the observation at any pressure point.

For an individual crossfloat balance, the effective area of the test gauge expressed in terms of the other experimental parameters is [13]:

$$
\begin{aligned}
A_{e(n)} & =A_{0}(1+b p)\left[1+\left(\alpha_{\mathrm{p}}+\alpha_{\mathrm{c}}\right)\left(T-T_{\mathrm{r}}\right)\right] \\
& =\frac{\sum_{i=1}^{n}\left[M_{\mathrm{i}}\left(1-\frac{\rho_{\mathrm{air}}}{\rho_{\mathrm{m}_{i}}}\right) \mathrm{g}\right]+\gamma_{\mathrm{f}} \cdot C}{p_{\mathrm{s}}+\Delta p}
\end{aligned}
$$

where

$M_{i} \quad$ is the true mass of the $i$ th weight on the test gauge

$\rho_{\text {air }}$ is the density of air in the vicinity of the weights

$\rho_{\mathrm{m}_{i}} \quad$ is the density of the $i$ th weight on the test gauge

$g \quad$ is the local acceleration due to gravity

$A_{0} \quad$ is the effective area at the reference temperature and atmospheric pressure

$b \quad$ is the pressure distortion coefficient of the piston and cylinder combination

$\alpha_{p} \quad$ is the linear thermal expansion coefficient of the piston

$\alpha_{c}$ is the linear thermal expansion coefficient of the cylinder

$T \quad$ is the temperature of the piston and cylinder

$T_{\mathrm{r}} \quad$ is the reference temperature

$\gamma_{\mathrm{f}}$ is the surface tension of the operating liquid

$C \quad$ is the circumference of the piston

$p_{\mathrm{s}} \quad$ is the pressure at the reference level of the standard gauge.

$\Delta p$ is the head correction $\left(\rho_{\mathrm{f}}-\rho_{\text {air }}\right) g H$, where $H$ is the height difference between the reference levels of the two gauges and $\rho_{\mathrm{f}}$ is the density of the pressure transmitting fluid. $\Delta p$ can be positive or negative depending on whether the reference level of the standard is lower or higher than that of the test gauge.
As it was not possible to bring the reference levels of the individual piston gauges to the same operating level during crossfloat, a pressure head correction term $(\Delta p)$ was applied. In this case, the reference level of NPL-28 was higher by $0.105 \mathrm{~m}$ than that of NIST- 45 .

A computer program developed and used at NIST [7] gives the effective area and the pressure coefficients of the test gauge based upon those of the standard. This program also provides the residuals and the standard deviation of the predicted value of the area, and the standard deviation of the coefficients.

\section{Results and Discussion}

Three test cycles, up to $26 \mathrm{MPa}$, were carried out during the intercomparison of NPL-28 and NIST-45. In one cycle, the pressure was increased to $(6,12,16,20$ and 26) MPa, and then decreased from (20 to 6) MPa in similar steps. In the other two cycles, the measurement proceeded from the highest pressure to the lowest and back to the highest. A fourth set of observations was also taken where the pressure was increased from the lowest to the highest pressure only. A total of 32 independent observations were made, nine in each of the first three test cycles and five in the fourth test.

Figure 4 shows a plot of the residuals of the effective area of NPL-28, as a function of the nominal applied pressure, when NPL-28 is crossfloated against NIST45. This figure gives the deviation of the measured values of the effective area, in parts in $10^{6}$, for the individual measured pressures, from the fitted equation $A_{\mathrm{e}}=A_{0}(1+b p)$ where $A_{0}=8.400 \quad 415 \times 10^{-5} \mathrm{~m}^{2}$ and $b=-2.05 \times 10^{-13} \mathrm{~Pa}^{-1}$. The distribution of the residuals of the effective area $\left(A_{c}\right)$ of NPL-28 in Fig. 4 is taken to be random.

The value of $A_{0}$ of NPL-28 as obtained by crossfloat against NPL- 100 exceeds by $1 \times 10^{-6}$ the value obtained by crossfloat against NIST-45. This $1 \times 10^{-6}$ difference is well below the $3 \sigma$ standard deviation of the $A_{0}$ coefficient, and hence the agreement at low pressure is excellent. Further, the value of $b$ for NPL-28 when it is crossfloated against NIST-45 differs from the value when it is crossfloated against NPL-100 (given in Table 1) by $0.43 \times 10^{-12} \mathrm{~Pa}^{-1}$. Considering the $3 \sigma$ standard deviations of these measured values, i.e., $0.2 \times 10^{-12}$ $\mathrm{Pa}^{-1}$ and $0.4 \times 10^{-12} \mathrm{~Pa}^{-1}$, respectively, the difference is not unreasonable. Additionally, these observed differences in $A_{0}$ and $b$ cause a relative difference in the effective area of $3.5 \times 10^{-6}$ at a measured pressure of 6 $\mathrm{MPa}$, increasing to $12 \times 10^{-6}$ at a full scale pressure of $26 \mathrm{MPa}$. These results are compatible with the measurement uncertainties associated with the individual piston gauges as given in Table 1. 


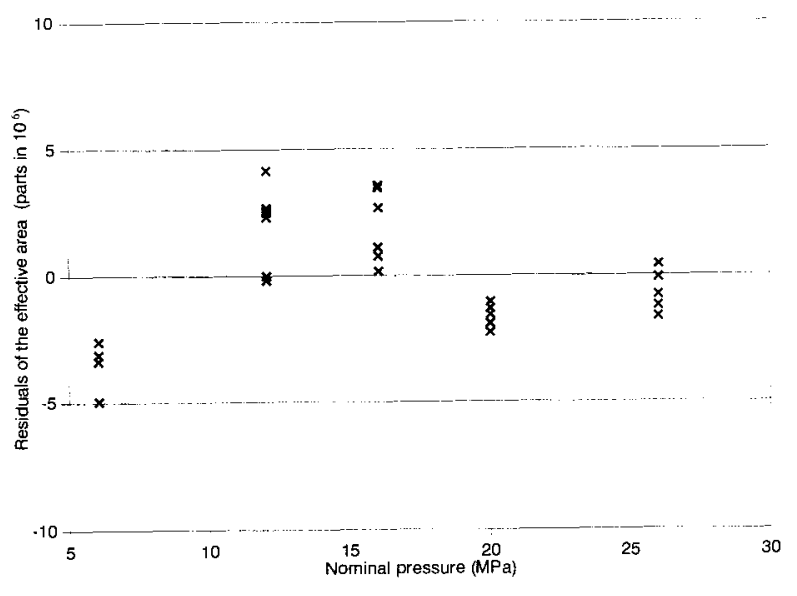

Fig. 4. Residuals of measured values of the effective area $\left(A_{\mathrm{e}}\right)$ of NPL-28 from the best linear fit of the model $A_{\mathrm{c}}=A_{0}(1+b p)$, where $A_{0}=8.400415 \times 10^{-5} \mathrm{~m}^{2}$ and $b=-2.05 \times 10^{-12} \mathrm{~Pa}^{-1}$, obtained when calibrated by NIST- 45 .

The uncertainty in the measurement of pressure using a piston gauge arises from two main sources: (1) inherent uncertainties associated with the gauge itself and (2) other uncertainties associated with the local experimental conditions. The former is mainly attributable to the determination of the effective area of the piston-cylinder assembly and uncertainties in the mass of the load and/ or piston. However, the latter arises from the experimental procedures, the major components of which were (i) uncertainty associated with the measurement of temperature, (ii) correction due to any difference of reference levels and (iii) the resolution of the balancing criteria when the two systems are in equilibrium.

During the cross float of NPL-28 and NIST-45 the fractional mass was adjusted so as not to contribute more than $\pm 1.2 \times 10^{-6}$ uncertainty $(3 \sigma)$ at the minimum pressure of $6 \mathrm{MPa}$, which decreases to less than $1 \times 10^{-6}$ at the full scale pressure of $26 \mathrm{MPa}$. As the reference levels were measured with an uncertainty of $5.00 \times 10^{-4}$ $\mathrm{m}(3 \sigma)$ and temperature was read with an estimated accuracy of $0.1{ }^{\circ} \mathrm{C}(3 \sigma)$, the contribution to the total estimated uncertainties in effective area due to temperature and difference in reference level is not significant compared to the total uncertainties associated with the standards NPL-28 and NIST-45

\section{Conclusions}

The comparison of the effective area of NPL-28 as determined by NIST-45 and NPL-100 show agreement between the two pressure standards (NPL-28 and NIST$45)$ that is significantly better than the estimated $(3 \sigma)$ uncertainty of either gauge. The low-pressure area of NPL- 28 obtained from NPL- 100 differs by only $1 \times 10^{-6}$ from the area value obtained during comparison with NIST -45 . The effective areas of NPL-28 determined by these same two paths differ by $3.5 \times 10^{-6}$ at $6 \mathrm{MPa}$, increasing to $12 \times 10^{-6}$ at $26 \mathrm{MPa}$. This study thus shows the agreement of measurements of effective area, and hence demonstrates the compatibility of the standards maintained by these two laboratories, for hydraulic pressures to $26 \mathrm{MPa}$.

\section{References}

[1] P. L. M. Heydemann and B. E. Welch, Experimental Thermodynamics, Volume II, B. LeNeindre and B. Vodar, eds., Butterworth \& Co. Ltd., London (1975), p. 147.

[2] P. L. M. Heydemann, C. R. Tilford, C. R. and R. W. Hyland, J. Vac. Sci. Technol. 14, 597 (1977).

[3] A. H. Bass, J. Phys. E: Sci. Instrum. 11, 682 (1978).

[4] K. Jain, C. Ehrlich, J. Houck, and J. K. N. Sharma, Meas. Sci. Technol. 4, 249 (1993).

[5] R. S. Dadson, S. L. Lewis, and G. N. Peggs, The Pressure Balance: Theory an Practice, National Physical Laboratory, Teddington, England, HMSO (1982).

[6] J. K. N. Sharma, K. K. Jain, A. K. Badyopadhyay, and J. Jāger, J. Phys. E: Sci. Instrum. 21, 635 (1988).

[7] J. C. Houck, G. F. Molinar, and R. Maghenzani, J. Res. Natl. Bur. Stand. (U.S.) 88, 253 (1983).

[8] J. C. Legras, G. F. Molinar, K. Schmalhofer, J. K. N. Sharma, M. Thane, and D. B. Walker, BIPM Int. Report 90/10 (1990)

[9] G. F. Molinar, L. Bianchi, J. K. N. Sharma, and K. K. Jain, High Temp. High Pressure 18, 241 (1986).

[10] H. Bauer and J. Jäger, Metrologia 16, 111 (1980).

[11] G. N. Peggs, S. L. Lewis, P. B. Clapham, J. Jäger, H. Bauer, G. Schopper, and N. Schultz, NPL Report No. M 32, (June 1978).

[12] G. N. Peggs, S. L. Lewis, and J. C. Legras, NPL Report No. M 39 (March 1980).

[13] K. Jain, C. Ehrlich, and J. Houck, Rev. Sci. Instrum. 63, 3127 (1992).

About the authors: Dr. J. K. N. Sharma is the recently retired Deputy Director and Head of Physico-Mechanical Standards at the National Physical Laboratory, New Delhi, India. Dr. Kamlesh Jain is a physicist in the Pressure Group at the National Physical Laboratory, New Delhi, India. Dr. Charles Ehrlich is the Leader of the Pressure Group in the Thermophysics Division of the National Institute of Standards and Technology. Mr. James Houck is a retired physicist from the Pressure Group in the Thermophysics Division of the National Institute of Standards and Technology. Mr. Donald Ward is a technician in the Pressure Group in the Thermophysics Division of the National Institute of Standards and Technology. The National Institute of Standards and Technology is an agency of the Technology Administration, U.S. Department of Commerce. 